\title{
EFEITO DA PRESSÃO DE TRABALHO E DE MODELOS DE PONTAS DE PULVERIZAÇÃO SOBRE A EFICIÊNCIA DE HERBICIDA DE CONTATO EM SOJA
}

\author{
WALTER BOLLER ${ }^{1}$, MARCOS MACHRY ${ }^{2}$
}

\begin{abstract}
RESUMO: Nos últimos anos, nas aplicações de herbicidas de contato em pós-emergência, tem-se verificado tendência de redução do volume de calda e a utilização de pontas de pulverização que operam com baixas pressões. Com o objetivo de avaliar pontas de pulverização de jatos planos, das séries Teejet XR, DG, TT e AI, operadas com três níveis de pressão de trabalho, foi conduzido um experimento visando ao controle de picão-preto (Bidens spp.), aplicando-se em pós-emergência da cultura da soja o herbicida de contato bentazon. As pontas de jatos planos das séries Teejet XR 110015, DG 110015 e TT 110015 foram operadas com pressões de 100; 200 e $300 \mathrm{kPa}$, e a ponta AI 110015, nas pressões de 200; 300 e $400 \mathrm{kPa}$. Avaliaram-se os níveis de controle das plantas de picão-preto aos 7; 14 e 21 dias após a aplicação dos tratamentos e a produtividade de grãos da cultura da soja. Observaram-se diferenças entre as pontas de pulverização e entre as pressões utilizadas. A ponta AI 110015 mostrou-se ineficiente para esse uso em todas as pressões avaliadas. A pressão de $100 \mathrm{kPa}$ mostrou-se inadequada para aplicações em pós-emergência de herbicida com ação de contato com as pontas XR, DG e TT. O aumento da pressão de pulverização reflete melhor controle das plantas daninhas com o herbicida utilizado.
\end{abstract}

PALAVRAS-CHAVE: tecnologia de aplicação, bentazon, Bidens spp.

\section{OPERATING PRESSURE AND SPRAY NOZZLES EFFECTS ON THE EFFICIENCY OF CONTACT HERBICIDE IN SOYBEANS}

\begin{abstract}
In recent years, contact herbicides have been sprayed, with reduced spray volumes, using spray nozzles that work at low pressures. To evaluate the flat fan spray nozzles of Teejet series XR, DG, TT and AI operated at different pressures, it was conducted an experiment for hairy beggar ticks (Bidens spp.) control. The experiment was carried out in Passo Fundo Municipality (Brazil), using bentazon contact herbicide in a soybean culture. The XR 110015, DG 110015 and TT 110015 nozzles were operated with pressure levels of 100; 200 and $300 \mathrm{kPa}$, and the AI 110015 nozzle with pressures of $200 ; 300$ and $400 \mathrm{kPa}$. It was evaluated the hairy beggar ticks control at 7; 14 and 21 days after treatment and the grain yield of soybean. The AI 110015 nozzle showed inappropriate for this herbicide application in all evaluated pressures. Pressure of $100 \mathrm{kPa}$ for the series XR, DG and TT, nozzles, were inadequate to use for contact post emergence herbicide application. All evaluated nozzles showed improved efficiency with the increase of pressure.
\end{abstract}

KEYWORDS: pesticides spray technology, bentazon, Bidens spp.

\section{INTRODUÇÃO}

Uma adequada aplicação de produto fitossanitário é aquela que, realizada no momento correto, proporciona cobertura suficiente do alvo e nele deposita a quantidade necessária de ingrediente ativo para eliminar ou abrandar, com segurança, um determinado problema, a fim de que sejam evitados danos econômicos (FUNDAÇÃO ABC, 1996). Para este autor, a cobertura do alvo indicada para herbicidas pós-emergentes de contato é de 30 a 40 gotas $\mathrm{cm}^{-2}$, e a segurança compreende o alojamento final do produto fitossanitário sobre o alvo da pulverização, evitando atingir animais, pessoas e áreas fora desse alvo, onde o produto pulverizado é indesejado.

\footnotetext{
${ }^{1}$ Eng 9 Agrônomo, Prof. Doutor, Faculdade de Agronomia e Medicina Veterinária, Universidade de Passo Fundo, Caixa Postal 611, Passo Fundo - RS, boller@upf.br

${ }^{2}$ Eng ${ }^{\mathrm{o}}$ Agrônomo, Prof. M. Sc., Faculdade Cantareira, São Paulo - SP, faculdade@ cantareira.br

Recebido pelo Conselho Editorial em: 16-11-2006
}

Aprovado pelo Conselho Editorial em: 3-10-2007 
O sucesso no controle químico de plantas daninhas depende de herbicidas eficazes, equipamentos e condições de ambiente adequadas, havendo muitos trabalhos com avaliações de herbicidas, porém poucas pesquisas se preocupam com as condições de ambiente e com o equipamento utilizado nas pulverizações (RUEDELL, 1995; 1999).

O volume de calda utilizado nas aplicações de produtos fitossanitários tem sido reduzido nos últimos anos. Tal fato deve-se, em grande parte, à utilização de herbicidas sistêmicos à base de glifosato, que apresentam melhor controle das plantas-alvo com volumes menores (próximos a $100 \mathrm{~L} \mathrm{ha}^{-1}$ ), comparados com volumes próximos a $200 \mathrm{~L} \mathrm{ha}^{-1}$ (THEISEN \& RUEDELL, 2004). Por comodidade, os usuários das máquinas aplicadoras costumam manter as mesmas regulagens (baixas pressões e baixos volumes de calda) que proporcionam os melhores resultados nas aplicações de herbicidas sistêmicos, para realizar as aplicações dos demais produtos fitossanitários. Também deve ser considerada, neste contexto, a evolução dos produtos fitossanitários e das pontas de pulverização. As pontas de pulverização de jatos planos atualmente disponíveis apresentam capacidade de abrir o ângulo do jato próximo do seu máximo, mantêm a distribuição uniforme das gotas sobre o alvo e produzem gotas de tamanho adequado, com baixas pressões de operação, possibilitando, dessa forma, a redução do volume de calda nas pulverizações agrícolas (SOUZA \& LHAMBY, 2000).

O uso de baixos volumes de pulverização permite o aproveitamento dos melhores horários de aplicação e torna-se possível com a utilização de pontas de jatos planos com faixa ampliada de pressão (série Teejet XR e similares), que podem operar com pressões de 100 a $130 \mathrm{kPa}$, aplicando volumes de 50 a $100 \mathrm{~L} \mathrm{ha}^{-1}$ (BIANCHI, 1998). Com as atuais técnicas de pulverização, é possível obter adequada cobertura e penetração da calda no dossel das culturas, com pontas específicas e gotas com diâmetro mediano volumétrico (DMV) adequado para cada situação (MAROCHI \& SCHMIDT, 1996).

As pontas de pulverização são os componentes mais importantes de um pulverizador, pois são responsáveis pela determinação da vazão da calda, pela distribuição uniforme do produto fitossanitário sobre o alvo biológico e pela formação das gotas de tamanho compatível com a finalidade a que se destinam (MATTHEWS, 1979). A utilização de pontas de jato plano com faixa de pressão ampliada permite aplicar quase todas as classes de produtos fitossanitários, uma vez que, com baixas pressões (100 a $130 \mathrm{kPa})$, produzem gotas de categorias mais grossas, mais resistentes à deriva e adequadas para herbicidas sistêmicos. Essas mesmas pontas, operadas com pressões maiores (até $400 \mathrm{kPa}$ ), geram gotas de categorias mais finas, proporcionando níveis de cobertura dos alvos compatíveis com as exigências de herbicidas de contato, inseticidas e fungicidas (SPRAYING SYSTEMS Co., 1999).

Tendo em vista a necessidade de evitar a contaminação ambiental pela deriva das pulverizações, os fabricantes de pontas de pulverização têm lançado no mercado pontas com significativo potencial antideriva, as quais produzem gotas de categorias mais grossas do que as pontas de jatos planos standard de mesma vazão, até quando operam com pressões de trabalho maiores (CUNHA et al., 2003). O mercado oferece pontas de jatos planos, que podem ser utilizadas com baixa pressão (100 a $300 \mathrm{kPa}$ ), que possibilitam aplicar baixos volumes de calda e reduzem a percentagem de gotas muito finas $(<100 \mu \mathrm{m})$ de 30 a $35 \%$ para menos de $10 \%$ (MAROCHI \& SCHMIDT, 1996). A redução da pressão de 400 para $200 \mathrm{kPa}$ resultou em menor percentagem do volume pulverizado composto por gotas menores que $100 \mu \mathrm{m}$ (CUNHA et al., 2003). Apesar da importância atribuída às pontas de pulverização utilizadas no resultado final de uma aplicação, muitas são as dúvidas a respeito do seu efeito.

O objetivo deste trabalho foi avaliar o efeito de quatro modelos de pontas de pulverização, quando operadas com três níveis de pressão, no controle químico de picão-preto em soja, com aplicação de herbicida pós-emergente com ação de contato. 


\section{MATERIAL E MÉTODOS}

O experimento foi conduzido na área experimental de herbologia da Faculdade de Agronomia e Medicina Veterinária, da Universidade de Passo Fundo, localizada no Município de Passo Fundo, na região fisiográfica do Planalto Médio do Rio Grande do Sul. As coordenadas geográficas da área são: altitude de $678 \mathrm{~m}$, latitude $28^{\circ} 15^{\prime} 46^{\prime} \mathrm{S}$ e longitude 52²4'24'W. A soja, cultivar Embrapa 66, foi semeada em área sob cobertura morta de $5.600 \mathrm{~kg} \mathrm{ha}^{-1}$ de aveia-preta, no dia 23-12-2000, com espaçamento de 0,45 m entre linhas, seguindo-se as indicações técnicas oficiais para essa cultura.

Foi utilizado o herbicida pós-emergente bentazon (Basagran), na dose de $720 \mathrm{~g}$ i.a. ha ${ }^{-1}$, aplicado no dia 15-1-2001, e como planta-alvo foi usado o picão-preto (Bidens spp.) no estádio de 2 a 4 folhas. Na ocasião, a soja estava no estádio V3 (terceiro nó visível e segundo trifólio aberto).

Utilizaram-se as pontas de pulverização de jatos planos das séries Teejet XR 110015, DG 110015, TT 110015, e as pontas de jato plano com indução de ar Teejet AI 110015, operadas segundo os níveis de pressões e gerando gotas de categorias que se observam na TABELA 1.

TABELA 1. Pontas de pulverização utilizadas, níveis de pressão e respectivas categorias das gotas, segundo informações do fabricante (SPRAYING SYSTEMS CO., 2006).

\begin{tabular}{lcc}
\hline Ponta de Pulverização & Pressão $(\mathrm{Kpa})$ & Categoria das Gotas* \\
\hline \multirow{2}{*}{ XR 110015} & 100 & Média \\
& 200 & Fina \\
& 300 & Fina \\
\hline \multirow{2}{*}{ DG 110015} & 100 & Grossa \\
& 200 & Média \\
TT 110015 & 300 & Média \\
\hline \multirow{3}{*}{ AI 110015} & 100 & Grossa \\
& 200 & Média \\
& 300 & Média \\
\hline
\end{tabular}

* Categorias de tamanho das gotas segundo norma ASAE S572 (ASAE, 2000).

As aplicações foram efetuadas com pulverizador costal, pressurizado com gás $\mathrm{CO}_{2}$. A barra de pulverização continha quatro pontas funcionais com espaçamento de $0,5 \mathrm{~m}$ e foi conduzida à altura de $0,5 \mathrm{~m}$ acima do alvo. O volume de calda foi mantido constante em $100 \mathrm{~L} \mathrm{ha}^{-1}$ para todos os tratamentos, alterando-se a velocidade de deslocamento, de acordo com a pressão de pulverização. No momento da aplicação dos tratamentos, a velocidade do vento variou de 0 a $4 \mathrm{~km}$ $\mathrm{h}^{-1}$, a temperatura do ar de 21 a $28^{\circ} \mathrm{C}$ e a umidade relativa do ar de 82 a $64 \%$. Ocorreu precipitação pluvial de dois milímetros, uma hora após o término das aplicações.

$\mathrm{O}$ delineamento experimental foi o inteiramente casualizado, com arranjo fatorial $(4 \times 3)$ e quatro repetições. As parcelas mediram 3,0 $\mathrm{m}$ de largura x 6,0 $\mathrm{m}$ de comprimento $\left(18 \mathrm{~m}^{2}\right)$.

As avaliações de controle de picão-preto foram realizadas aos 7; 14 e 21 dias após a aplicação dos tratamentos. Para avaliação do controle, foi realizada pré-contagem das plantas existentes no dia da aplicação dos tratamentos em cada parcela, demarcando-se os pontos das amostragens. Essa contagem foi efetuada em duas amostras de $0,5 \mathrm{~m}^{2}(1,0 \mathrm{~m} \times 0,5 \mathrm{~m})$ por parcela, e nas avaliações subseqüentes foram efetuadas contagens de plantas vivas nessas mesmas subáreas amostradas. Calculou-se, então, a percentagem de controle em relação à pré-contagem, em que $100 \%$ de controle foi ausência de plantas daninhas e 0\% de controle foi presença do mesmo número de plantas daninhas encontrado na pré-contagem. Para a avaliação da produtividade de grãos, foi efetuada a colheita com colhedora de parcelas, colhendo-se as três linhas centrais de cada 
parcela, no comprimento de 4,0 $\mathrm{m}$, perfazendo área colhida de 5,4 $\mathrm{m}^{2}$. Os resultados foram submetidos à análise de variância e quando o teste $\mathrm{F}$ foi significativo a $5 \%$, compararam-se as médias por meio do teste de Tukey, a 5\% de probabilidade (BANZATO \& KRONKA, 1995).

\section{RESULTADOS E DISCUSSÃO}

A pré-contagem revelou população média de 19 plantas $\mathrm{m}^{-2}$ de picão-preto, com variação de 5 a 39 plantas $\mathrm{m}^{-2}$. Nas avaliações de controle do picão-preto realizadas aos 7; 14 e 21 dias após a aplicação (Tabela 2), foram observadas diferenças estatísticas para os dois fatores analisados. Nas três avaliações, a aplicação do herbicida com as pontas da série AI 110015 (gotas de categorias muito grossa até extremamente grossa) proporcionou níveis de controle de picão-preto inferior, comparado com as aplicações realizadas com as demais pontas. Já, entre as pontas XR 110015, DG 110115 e TT 110015, não houve diferenças estatísticas. Em relação ao fator pressão de trabalho, para todas as pontas avaliadas, a pressão mais baixa mostrou-se inferior às demais, embora a ponta XR 110015 produza gotas de categoria média na pressão de $100 \mathrm{kPa}$ e gotas finas nas pressões de 200 e $300 \mathrm{kPa}$, enquanto as pontas DG 110015 e TT 110015 produzem gotas grossas à pressão de $100 \mathrm{kPa}$ e médias nas demais. Em parte, esse comportamento pode ser atribuído à redução do tamanho das gotas e ao aumento da cobertura do alvo ocasionado pelo aumento da pressão de trabalho, conforme relato de CUNHA et al. (2003). Outra possível causa é que, à pressão mais baixa $(100 \mathrm{kPa})$, as pontas de energia hidráulica, via-de-regra, ainda não conseguem abrir completamente o ângulo do jato que produzem (FREITAS et al., 2005), resultando distribuição irregular.

TABELA 2. Percentagem de controle de picão-preto em manejo químico com herbicida de contato bentazon, em função de diferentes pontas de pulverização e pressões de trabalho aos 7; 14 e 21 dias após a aplicação dos tratamentos (DAT), e produtividade de grãos $\left(\mathrm{kg} \mathrm{ha}^{-1}\right)$ da cultura da soja.

\begin{tabular}{|c|c|c|c|c|}
\hline \multirow{2}{*}{ Fator } & \multicolumn{3}{|c|}{ Avaliações } & \multirow{2}{*}{$\begin{array}{l}\text { Produtividade de } \\
\text { grãos }\left(\mathrm{kg} \mathrm{ha}^{-1}\right) * *\end{array}$} \\
\hline & $7 \mathrm{DAT}^{*}$ & $14 \mathrm{DAT}^{*}$ & $21 \mathrm{DAT} *$ & \\
\hline \multicolumn{5}{|l|}{ Ponta } \\
\hline XR 110015 & 68,9 a & $62,5 \mathrm{a}$ & $62,2 \mathrm{a}$ & 2.511 \\
\hline DG 110015 & $62,6 \mathrm{a}$ & 59,5 a & $59,4 \mathrm{a}$ & 2.332 \\
\hline TT 110015 & $66,7 \mathrm{a}$ & $62,5 \mathrm{a}$ & $62,1 \mathrm{a}$ & 2.372 \\
\hline AI 110015 & $46,8 \mathrm{~b}$ & $41,6 \mathrm{~b}$ & $37,1 \mathrm{~b}$ & 2.209 \\
\hline \multicolumn{5}{|c|}{ Pressão $(\mathrm{kPa}) * * *$} \\
\hline 100 & $50,8 \mathrm{~b}$ & $48,1 \mathrm{~b}$ & $45,1 \mathrm{~b}$ & 2.220 \\
\hline 200 & $64,1 \mathrm{a}$ & $59,5 \mathrm{a}$ & $58,7 \mathrm{a}$ & 2.405 \\
\hline 300 & $68,8 \mathrm{a}$ & $61,1 \mathrm{a}$ & $61,1 \mathrm{a}$ & 2.443 \\
\hline C.V. (\%) & 15,5 & 23,3 & 18,8 & 12,1 \\
\hline
\end{tabular}

Esses resultados não concordam com os relatos de ETHERIDGE et al. (2001), que utilizaram pressão de trabalho de $267 \mathrm{kPa}$ e obtiveram níveis de controle de plantas daninhas semelhantes entre pontas das séries XR e AI, em aplicações dos herbicidas glyphosate, glufosinate de amônio e paraquat. Por outro lado, os resultados concordam parcialmente com aqueles de JENSEN et al. (2001), que obtiveram diferenças na eficácia biológica de herbicidas de contato quando esses foram aplicados com pontas de pulverização de jato plano de baixa deriva, pontas de jato plano duplo e pontas convencionais. Esses níveis de controle de picão-preto (entre 60 e 80\%) são considerados médios (REUNIÃO DE PESQUISA DE SOJA DA REGIÃO SUL, 2005). 
Considerando que o herbicida utilizado é eficaz contra a espécie daninha utilizada, esperavam-se níveis de controle muito próximos a 100\%. Especula-se que uma das possíveis causas para os níveis de controle não se aproximarem de $100 \%$ pode ter sido a emergência de novas plantas de picão-preto logo após a aplicação dos tratamentos, uma vez que o herbicida utilizado apresenta apenas ação de contato em pós-emergência, não atuando sobre as sementes no solo.

Por sua vez, a produtividade de grãos da soja não apresentou diferenças significativas, a 5\% de probabilidade, entre os tratamentos, o que pode ser creditado, parcialmente, à infestação inicial de plantas de picão-preto, que foi considerada de baixa a média e ao retardamento do estabelecimento dessas ocasionado pela presença da palhada de aveia-preta, conferindo vantagem à cultura.

\section{CONCLUSÕES}

Os resultados obtidos permitem concluir que, para as condições do experimento, existem diferenças entre os níveis de controle de picão-preto com o herbicida pós-emergente de contato bentazon, quando esse é aplicado com as diferentes pontas de pulverização avaliadas.

O aumento da pressão de pulverização na aplicação do herbicida pós-emergente de contato bentazon aumenta a eficácia do controle das plantas daninhas.

As pontas AI 110015 são impróprias para a aplicação do herbicida pós-emergente de contato bentazon, independentemente da pressão de pulverização utilizada.

\section{REFERENCIAS}

ASAE. AMERICAN SOCIETY OF AGRICULTURAL ENGINEERING. S572. Spray nozzle classification by droplet spectra. In: ASAE Standard, AUG99. St. Joseph, 2000. p.389-91.

BANZATO, D.A.; KRONKA, S.N. Experimentação agrícola. 3.ed. Jaboticabal: FUNEP, 1995. $274 \mathrm{p}$.

BIANCHI, M.A. Manejo integrado de plantas daninhas. In: SILVA, M.T.B. A soja em rotação de culturas no plantio direto. Cruz Alta: FUNDACEP FECOTRIGO/SENAR, 1998. cap.6, p.157-85.

CUNHA, J.P.A.R.; TEIXEIRA, M.M.; COURY, J.R.; FERREIRA, L.R. Avaliação de estratégias para redução da deriva de agrotóxicos em pulverizações hidráulicas. Planta Daninha, Viçosa, v.21, n.2, p.325-32, 2003.

ETHERIDGE, R.E.; HART, W.E.; HAYES, R.M.; MUELLER, T.C. Effect of venturi-type nozzles and application volumes on postemergence herbicide efficacy. Weed Technology, Champaign, v.15, n.1, p.75-80, 2001.

FREITAS, F.C.L.; TEIXEIRA, M.M.; FERREIRA, L.R.; MACHADO, A.F.L.; VIANA, R.G. Distribuição volumétrica de pontas de pulverização turbo-teejet 11002 em diferentes condições operacionais. Planta Daninha, Viçosa, v.23, n.1, p.161-7, 2005.

FUNDAÇÃO ABC. Conquiste o futuro com a tecnologia presente: tecnologia de aplicação de defensivos. Castro: Fundação ABC, 1996. 36 p.

JENSEN, P.K.; JÖRGENSEN, L.N.; KIRKNEL, E. Biological efficacy of herbicides and fungicides applied with low-drift and twin-fluid nozzles. Crop Protection, Guildford, v.20, n.1, p.57-64, 2001.

MAROCHI, A. I.; SCHMIDT, W. Plantio direto na palha: tecnologia de aplicação e uso de Scorpion no sistema. Castro: Fundação ABC-DowElanco, 1996. 43 p.

MATTHEWS, G. A. Pesticide application methods. London: Longman, 1979. 334 p. 
REUNIÃO DE PESQUISA DE SOJA DA REGIÃO SUL. Indicações técnicas para a cultura da soja no Rio Grande do Sul e em Santa Catarina 2005/2006. Passo Fundo: Faculdade de Agronomia e Medicina Veterinária, 2005. 157 p.

RUEDELL, J. Tendências e estratégias na tecnologia de aplicação de herbicidas. In: CONGRESSO BRASILEIRO DE CIÊNCIA DAS PLANTAS DANINHAS, 20., 1995, Florianópolis. Palestras... Florianópolis: SBHED/UFSC, 1995. p.67-9.

RUEDELL, J. Controle de plantas daninhas. In: MATZENBACHER, R.G. A cultura da aveia no sistema de plantio direto. Cruz Alta: FUNDACEP FECOTRIGO/SENAR, 1999. p.85-119.

SOUZA, R.O. de; LHAMBY, J.C.B. Influência do volume de calda e de bicos de pulverização no manejo de azevém. Passo Fundo: EMBRAPA TRIGO/Projeto METAS, 2000. 20 p.

SPRAYING SYSTEMS CO. Produtos de pulverização para agricultura - Catálogo 46M-BR/P. Diadema, 1999. 104 p.

SPRAYING SYSTEMS CO. Teejet technologies - Catálog 50M. Wheaton, 2006. 192 p.

THEISEN, G.; RUEDELL, J. Tecnologia de aplicação de herbicidas: teoria e prática. Passo Fundo: Aldeia Norte, 2004. 90 p. 